\title{
On the Question of Defining the Association Constants by the Method of Fluorescence Quenching
}

\author{
Nikolay L. Lavrik, Nikolay M. Bazhin \\ Voevodsky Institute of Chemical Kinetics and Combustion SB RAS, Novosibirsk, Russian Federation \\ Email: lavrik@kinetics.nsc.ru
}

Received 5 September 2014; revised 20 October 2014; accepted 5 November 2014

Copyright (C) 2014 by authors and Scientific Research Publishing Inc.

This work is licensed under the Creative Commons Attribution International License (CC BY).

http://creativecommons.org/licenses/by/4.0/

cc) (i)

\section{Abstract}

A study is made on the previously ignored problem of the dependence of a static fluorescence quenching Stern-Volmer constant $K_{\mathrm{sv}}$ on the initial concentration of $[\mathrm{F}]_{0}$ fluorophore $F$. This correlation is shown to exist. It is concluded that the Stern-Volmer quenching constant may be used as association constant $K$ only with $K \cdot[\mathrm{F}]_{0} \ll 1$.

\section{Keywords}

Fluorescence Quenching, Association Constants, Stern-Volmer Constant

\section{Introduction}

Determination of the association constants $K$ is one of the most common tasks of physical chemistry, biochemistry, chemistry, etc. The constant $K$ in Equation (1) is taken as the association constant

$$
K=\frac{\mathrm{F}+\mathrm{Q} \leftrightarrow \mathrm{FQ} \quad K,}{\left([\mathrm{~F}]_{0}-[\mathrm{FQ}]\right)\left([\mathrm{Q}]_{0}-[\mathrm{FQ}]\right)} .
$$

In (1) and (2), F and $Q$ are the complexing reagents; FQ is the complex of reagents; $[F]_{0}$ and $[\mathrm{Q}]_{0}$ are the initial concentrations of $\mathrm{F}$ and $\mathrm{Q}$ according to preparation.

From Equation (2) it easily follows [1] that the relative concentration $(y)$ of complex FQ can be calculated 
from the equation

$$
y=\frac{[\mathrm{FQ}]}{[\mathrm{F}]_{0}}=\frac{(1+p+q)}{2} \cdot\left(1-\sqrt{1-\frac{4 p}{(1+p+q)^{2}}}\right)
$$

where

$$
p=[\mathrm{Q}]_{0} /[\mathrm{F}]_{0}, \quad q=1 /\left(K \cdot[\mathrm{F}]_{0}\right) .
$$

One of the numerous methods to determine the value of $K$ is the fluorescence one [2]. The basis of the described method is the assumption that the complexes FQ do not fluoresce (static quenching) and that the dynamic quenching of excited molecules $\mathrm{F}$ is absent.

The essence of this approach is that the fluorescence quenching constant $K_{\mathrm{SV}}$ is estimated from the Stern-Volmer equation

$$
I_{0} / I=1+K_{\mathrm{SV}} \cdot[\mathrm{Q}]_{0} .
$$

In (3), $I_{0}$ and $I$ are the fluorescence intensities of the fluorophore $\mathrm{F}$ in the absence and in the presence of quencher Q. The resulting constant $K_{\mathrm{SV}}$ is taken as the $K$ value [2]

$$
K_{\mathrm{SV}}=K \text {. }
$$

However, in the case of static quenching, the constant $K_{\mathrm{sV}}$ depends on the initial concentration of the fluorophore. This factor does not take into account usually [2]. The cause of such dependence is the formation of complexes FQ, which leads to a decrease in the concentration of free molecules $\mathrm{F}$ in contrast to the usual practice of using Stern-Volmer equation in the systems in which the concentration of the fluorophore does not depend on the concentration of the quencher.

The present work is devoted to the extent of correction to the experimental Stern-Volmer constant $K_{\mathrm{sv}}$ for the case of static fluorescence quenching under continuous illumination.

\section{Fluorescence Quenching under the Conditions of Nonfluorescent Complex Formation}

Consider now luminescence quenching in the presence of complex formation under stationary excitation. The following scheme holds for this case:

$$
\begin{array}{lc}
\mathrm{F}+\mathrm{Q} \leftrightarrow \mathrm{FQ} & K, \\
\mathrm{~F}+h v \rightarrow \mathrm{F}^{*} & \alpha I_{e x}, \\
\mathrm{~F}^{*} \rightarrow h v+\mathrm{F} & k_{1}, \\
\mathrm{~F}^{*} \rightarrow \mathrm{F} & k_{2} .
\end{array}
$$

In (7)-(9), $\alpha$ is the coefficient depending on experimental conditions and molar absorption coefficient of fluorofor, $I_{e x}$ is the intensity of exciting illumination, $k_{1}$ and $k_{2}$ are the radiative and non-radiative constants of the excited electronic states of fluorophore $\mathrm{F}^{*}$, respectively, and the $K$ value is the same as that in (1). From (7)-(9), we get

$$
\frac{\mathrm{d}\left[\mathrm{F}^{*}\right]}{\mathrm{d} t}=\alpha I_{e x}[\mathrm{~F}]-\left(k_{1}+k_{2}\right)\left[\mathrm{F}^{*}\right]=0 .
$$

Thus, in general, from (10) the fluorescence intensity $I$ is of the form

$$
I=k_{1}\left[\mathrm{~F}^{*}\right]=\alpha I_{e x} \frac{k_{1}}{\left(k_{1}+k_{2}\right)}[\mathrm{F}] .
$$

However, for a particular case of $[\mathrm{Q}]_{0}=0$, the fluorescence intensity is 


$$
I_{0}=k_{1}\left[\mathrm{~F}^{*}\right]=\alpha I_{e x} \frac{k_{1}}{\left(k_{1}+k_{2}\right)}[\mathrm{F}]_{0} .
$$

In fluorescence quenching the $I_{0} / I$ value is used. From (10)-(12) we obtain

$$
I_{0} / I=[\mathrm{F}]_{0} /[\mathrm{F}]
$$

Taking into account that

$$
[\mathrm{F}]=[\mathrm{F}]_{0}-y[\mathrm{~F}]_{0}
$$

we get either

$$
\frac{I_{0}}{I}=\frac{[\mathrm{F}]_{0}}{[\mathrm{~F}]}=\frac{1}{1-y}
$$

or

$$
\frac{I_{0}}{I}=\frac{1}{1-\frac{(1+p+q)}{2} \cdot\left(1-\sqrt{1-\frac{4 p}{(1+p+q)^{2}}}\right)} .
$$

For the small values of $p$, we have

$$
\frac{I_{0}}{I} \approx 1+\frac{p}{1+q} .
$$

To determine the Stern-Volmer constants, $\frac{I_{0}}{I}$ is measured as a function of $[\mathrm{Q}]_{0}$ (the dependence of $\frac{I_{0}}{I}$ on $[\mathrm{Q}]_{0}$ ). For this dependence with small $[\mathrm{Q}]_{0}$ values, we get

$$
\frac{I_{0}}{I} \approx 1+\frac{p}{1+q}=1+\frac{K \cdot[\mathrm{Q}]_{0}}{1+K \cdot[\mathrm{F}]_{0}}
$$

Comparing (18) with (6) indicates that in the case of complex formation, the Stern-Volmer quenching constant $K_{\mathrm{sv}}$ is not equal to $K$,

$$
K_{\mathrm{SV}}=\frac{K}{1+K \cdot[\mathrm{F}]_{0}} .
$$

Thus, $\frac{K}{1+K \cdot[\mathrm{F}]_{0}}$ rather than $K$ is measured experimentally. The latter is usually associated with a complex constant. This correction to unity in the denominator of Equation (19), equal to $K \cdot[\mathrm{F}]_{0}$ takes into account the fact that the concentration of molecules $\mathrm{F}$ is less than $[\mathrm{F}]_{0}$ because of complex formation. Thus, the generally accepted equating of the experimental quenching constant $K_{\mathrm{sv}}$ to the equilibrium one (complexing) $K$ [2] is valid only if $K \cdot[\mathrm{F}]_{0} \ll 1$.

The value of $K$ can be estimated from the dependence of $K_{\mathrm{sV}}^{-1}$ on $[\mathrm{F}]_{0}$

$$
1 / K_{\mathrm{SV}}=1 / K+[\mathrm{F}]_{0} \text {. }
$$

The $1 / K$ value and then, respectively, that of $K$ are determined from the cut on the Y-axis.

\section{Conclusion}

It is concluded then that introducing the above correction may be a key moment in obtaining the true values of the association constants. The relation $K \cdot[\mathrm{F}]_{0} \ll 1$ may fail in the case of reagents (fluorophores) with a low 
quantum yield. In this case, the high concentrations of $[\mathrm{F}]_{0}$ are used to provide reliable observations. This necessitates the introduction of a corrective factor which is also obligatory for the case of large complexing constants.

\section{References}

[1] De Weert, M. and Stella, L. (2011) Fluorescence Quenching and Ligand Binding: A Critical Discussion of a Popular Methodology. Journal of Molecular Structure, 998, 144-150. http://dx.doi.org/10.1016/j.molstruc.2011.05.023

[2] Lakovicz, J. (2010) Principles of Fluorescence Spectroscopy. 3rd Edition, Springer, Berlin. 
Scientific Research Publishing (SCIRP) is one of the largest Open Access journal publishers. It is currently publishing more than 200 open access, online, peer-reviewed journals covering a wide range of academic disciplines. SCIRP serves the worldwide academic communities and contributes to the progress and application of science with its publication.

Other selected journals from SCIRP are listed as below. Submit your manuscript to us via either submit@scirp.org or Online Submission Portal.
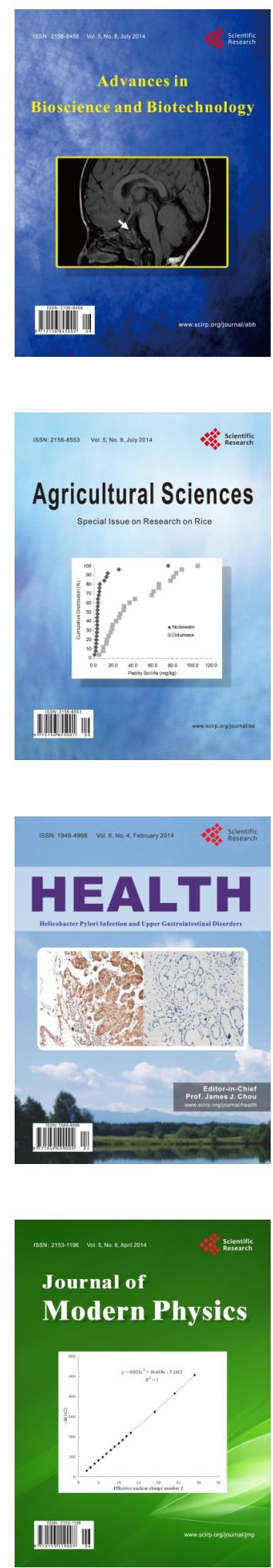
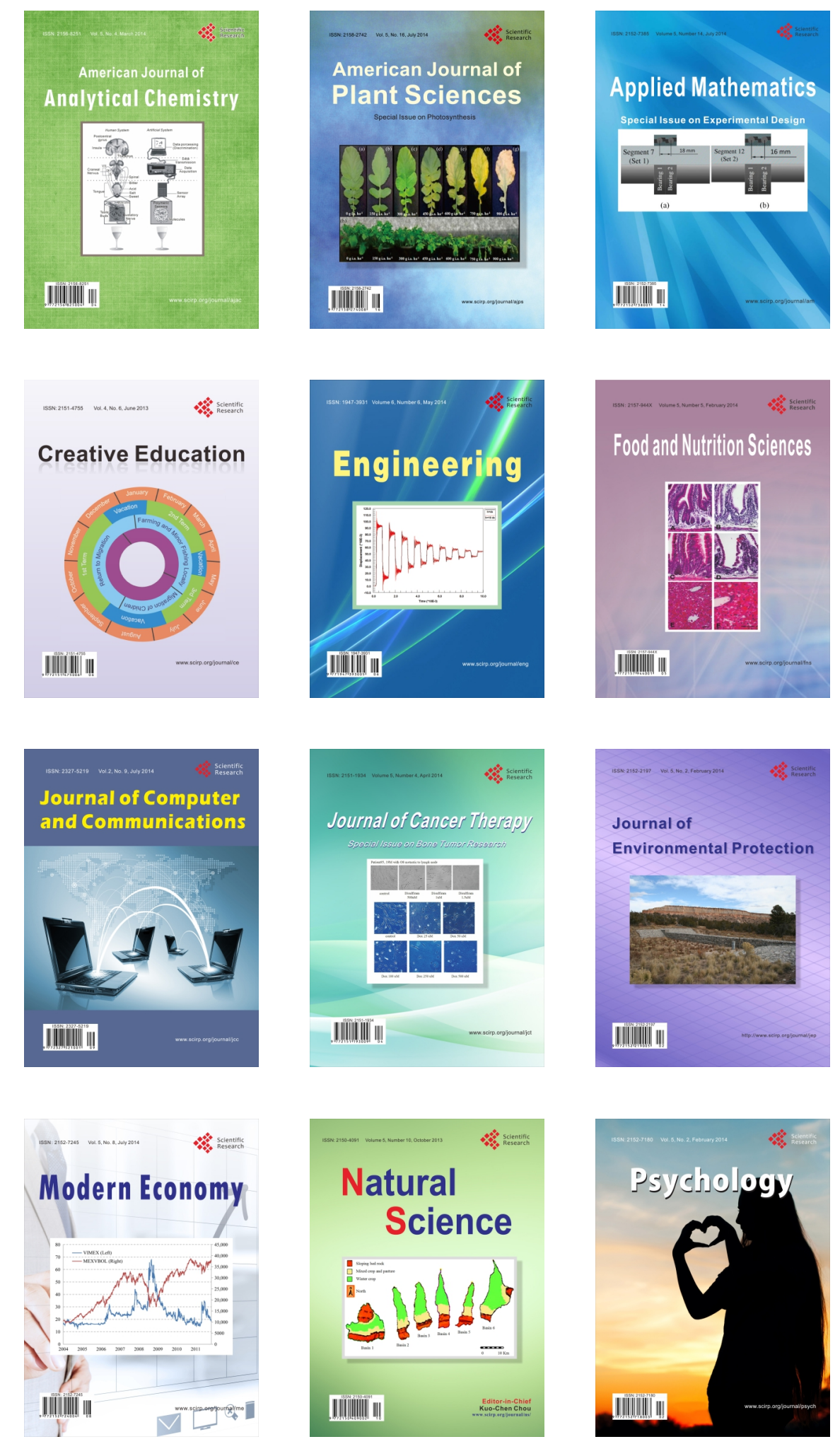